\title{
El Diseño Basado en Prestaciones frente al Incendio de las Estructuras de Edificación
}

\section{Fire Performance-Based Design of Building Structures}

\author{
$\underline{\text { C. Muñoz Blanc }}^{(*)}$, I. Fortea Navarro ${ }^{(*)}$
}

\section{RESUMEN}

En el presente trabajo se presentan las ventajas de utilizar el Diseño Basado en Prestaciones cuando se afronta la seguridad de las estructuras frente a la acción del incendio, se aporta una visión general del método y se muestran las ventajas de su aplicación en proyectos singulares de edificación a través de un caso real de estudio. Aunque el diseño prestacional tiene un amplio recorrido en países como el Reino Unido, Estados Unidos o Japón, con más de 30 años de evolución y desarrollo, en España hace apenas una década que la normativa ha abierto las puertas a utilizar el diseño prestacional. Sin embargo, aunque el Código Técnico de la Edificación anima a los diseñadores a llevar a cabo esta estrategia de trabajo, aún es poca la información que incorpora para tal fin.

Palabras clave: Diseño Basado en Prestaciones; fuego real, dinámica de fluidos; riesgos de incendio; modelos termomecánicos.

\section{ABSTRACT}

This Paper presents the advantages of Performance-Based Design regarding safety of structures under fire action. A general vision of this method is provided, and the advantages of its application are shown through a real case study. Although Performance Based Design has a long career in countries such as United Kingdom, United States or Japan, with more than 30 years of development and evolution, in Spain the code has allowed to use this method since just a decade ago. Nevertheless, although Spanish Building Technical Code encourages designers to carry out this work strategy, the information that is included for this purpose is yet scarce.

Keywords: Performance-Based Design; real fire; fluid dynamics; fire risk; thermo-mechanical models.

(*) Universitat Politècnica de Catalunya. E.T.S. Arquitectura de Barcelona. Departamento de Tecnología en la Arquitectura. Barcelona (España).

Persona de contacto/Corresponding author: carlos.munoz@upc.edu (C. Muñoz Blanc)

ORCID: http://orcid.org/oooo-0002-8996-1371 (C. Muñoz Blanc);

http://orcid.org/oooo-0002-0016-6073 (I. Fortea Navarro).

Cómo citar este artículo/Citation: Muñoz Blanc. C., Fortea Navarro, I. (2017). El Diseño Basado en Prestaciones frente al Incendio de las Estructuras de Edificación. Informes de la Construcción, 69(547): e215, doi: http://dx.doi.org/10.3989/id56471.

Copyright: (C) 2017 CSIC. Licencia / License: Salvo indicación contraria, todos los contenidos de la edición electrónica de Informes de la Construcción se distribuyen bajo una licencia de uso y distribución Creative Commons Attribution License (CC BY) Spain 3.o. 


\section{INTRODUCCIÓN}

Actualmente en España existen dos metodologías de aproximación al diseño de las estructuras cuando se afronta la seguridad en caso de incendio (1) (2): la vía prescriptiva y los métodos basados en prestaciones. Mientras que el Diseño Basado en Prestaciones se basa en el planteamiento de unas medidas de protección particularizadas a cada edificio, en base al conocimiento relativo al desarrollo que tendrá el incendio en cada escenario probable, tradicionalmente han sido las reglas prescriptivas las que han predominado en la concepción de cualquier tipo de edificio. En estas últimas la adopción de una curva normalizada temperatura-tiempo [1] sirve para determinar unos niveles mínimos de resistencia de los elementos estructurales. Estos elementos se verifican de manera individualizada en los hornos de ensayo, pero no se reproducen las condiciones reales de los incendios en espacios cerrados. A pesar de que esto no deja de ser una simplificación, una adecuada adopción de esta curva normalizada ha evitado en numerosas ocasiones el colapso de las estructuras de edificación durante la evacuación de los inmuebles y la actuación de los equipos de emergencia.

$$
\theta_{g}=\theta_{\mathrm{o}}+345 \cdot \log _{10}(8 \cdot t+1)
$$

Sin embargo, el uso de esta curva normalizada deriva en un gran número de ocasiones en un sobrecoste innecesario en la construcción, como consecuencia de un sobredimensionamiento de los materiales de protección o un uso irracional de los mismos para dar respuesta a una acción accidental de muy baja probabilidad de suceso. Como contrapartida, en un reducido número de construcciones el uso de la curva normalizada infravalora las temperaturas máximas que se pueden llegar a alcanzar en una estructura.

Estudios estadísticos realizados en el National Institute of Standards and Technology (NIST) indican que aproximadamente dos terceras partes de las víctimas de los incendios se producen debido a la intoxicación por humo después de producirse la combustión súbita generalizada y, en muchas ocasiones, en estancias diferentes al origen del fuego (3), es decir, no por las elevadas temperaturas o por el colapso de las estructuras.

Además, se debe tomar conciencia en relación a las limitaciones que un diseño estructural basado en criterios prescriptivos tendrá sobre el edificio. Un método basado en una curva ficticia que evalúa la resistencia de los elementos estructurales de manera individual no puede determinar la capacidad de un entramado resistente de redistribuir los esfuerzos cuando ciertos componentes del conjunto se ven debilitados por la acción del fuego. En el lado opuesto, los métodos prescriptivos no son capaces de determinar los esfuerzos térmicos que se producen en las estructuras de carácter indeterminado, debido a la coacción que las mismas padecen frente a la dilatación de sus elementos individuales cuando están sometidas a un incremento térmico. Un caso real de la poca idoneidad de los métodos prescriptivos en algunas ocasiones se incluye en el apartado 6 del presente trabajo.

\section{EL DISEÑO BASADO EN PRESTACIONES}

Uno de los objetivos fundamentales del Diseño Basado en Prestaciones es poder plantear las medidas de protección más adecuadas para cada edificio en particular. En el caso del diseño de las estructuras, éste se realiza a partir del conocimiento de las curvas temperatura-tiempo reales que afectarán a los entramados resistentes.

Desde hace décadas uno de los problemas fundamentales de todo el proceso de análisis de las estructuras en caso de incendio ha pasado por determinar en primer lugar las leyes temperatura-tiempo reales que se deben considerar en cada edificio particular y, una vez obtenidas, establecer posteriormente una relación con la curva normalizada ISO-834 que permita dictaminar unas medidas activas y pasivas de protección, ensayadas según parámetros normalizados.

El Diseño Basado en Prestaciones es único para cada edificio analizado mediante esta metodología. Sin embargo, se puede establecer una metodología de trabajo estructurada y aplicable a cualquier estudio prestacional (Figura 1) (4).

El análisis de los riesgos del incendio, incluido en la fase inicial del Diseño Basado en Prestaciones, habitualmente se desarrolla mediante una evaluación probabilística del riesgo, asignando a cada riesgo generado según la actividad una probabilidad de ocurrencia y una gravedad de daños ocasionados. Para ello se genera una matriz en la que se ubican los diferentes riesgos y se les asigna un índice de riesgo global. Tal y como se puede observar en la Figura 2, en la matriz se sitúan los diferentes riesgos de incendio, los cuales se pueden definir, por ejemplo, como Ro1, Ro2, etc. A cada probabilidad de ocurrencia del incendio se le asigna un valor numérico, variable del 1 (mínimo riesgo) al 6 (máximo riesgo). Lo mismo se realiza con las consecuencias, también numeradas del 1 al 5 , según el daño probable derivado de cada incendio. A cada riesgo se le asigna un valor numérico, resultado del producto de ambos números. Serán aquellos riesgos con un índice de riesgo global más severo (asociados en la matriz a los colores cálidos más intensos) los que se considerarán más desfavorables, por causar unas mayores pérdidas económicas y/o humanas. Son estos los que deberán ser evaluados en el análisis prestacional, estableciendo en cada proyecto particular cuál debe ser el índice de riesgo global a partir del que se deba considerar cada escenario.

Durante la fase de desarrollo del análisis prestacional de una estructura de edificación frente a la acción del incendio se pueden establecer tres pilares fundamentales que, complementándose entre ellos, permitirán verificar el cumplimiento de unos objetivos y dictaminar sobre la integridad de cualquier entramado resistente. Estos pilares son la simulación real de los incendios, el estudio de la transferencia de calor a los elementos estructurales y el análisis termo-mecánico de la estructura. Un amplio conocimiento de la física del problema que se encierra en cada uno de ellos debe permitir afrontar el Diseño Basado en Prestaciones con unos criterios de análisis y unos objetivos de diseño que garanticen la integridad de la estructura durante todo el desarrollo del incendio.

\section{OBJETIVOS DEL DISEÑO BASADO EN PRESTACIONES}

En un momento de la historia de la Arquitectura en que cada vez se promueve más el desarrollo de edificios tecnológicamente innovadores, el Diseño Basado en Prestaciones frente a la acción del fuego tiene unas claras ventajas respecto de las reglas prescriptivas. 


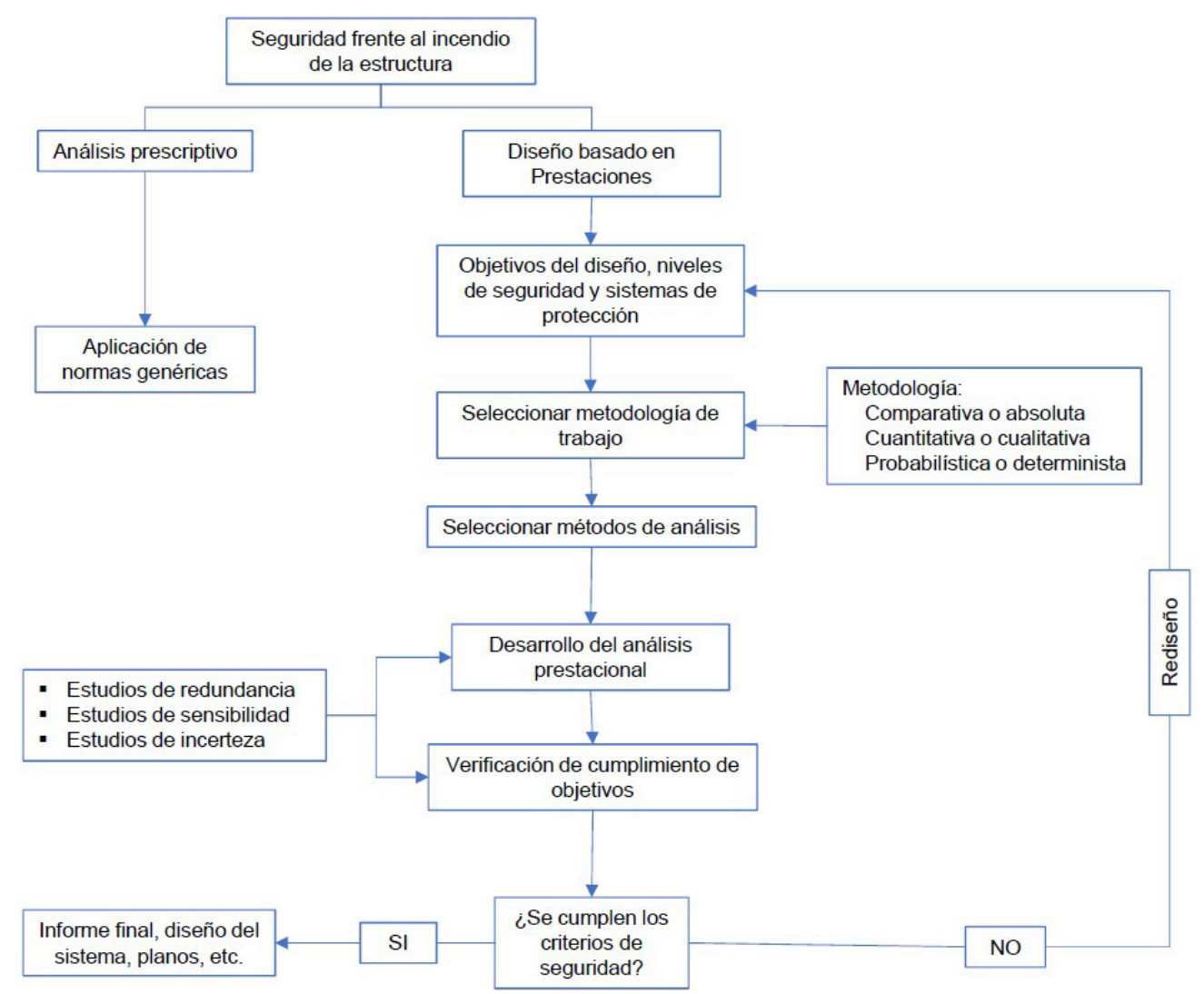

Figura 1. Metodología de análisis del Diseño Basado en Prestaciones.

En primer lugar, un Diseño Basado en Prestaciones permite conocer cuál será la respuesta real que tendrá la estructura frente a la acción del fuego, mientras que unas reglas prescriptivas únicamente establecen unos criterios genéricos que, como tales, deben cubrir un amplio espectro de estructuras y de tipologías de edificios, siendo incapaces de caracterizar el comportamiento real de un sistema estructural concreto.

En segundo lugar, el Diseño Basado en Prestaciones aporta una flexibilidad en el diseño, dado que lo que se deben cumplir son unos objetivos concretos de comportamiento y de seguridad de la estructura frente a unas acciones térmicas accidentales, particularizadas a la realidad de cada edificio.

En tercer lugar, la evolución normativa en las últimas décadas ha promovido que el grado de exigencia en la seguridad haya sido cada vez mayor. Lograr estos estándares de manera prescriptiva obliga, en edificios singulares, a unos costes económicos que muchas veces son difícilmente asumibles por el proyecto. Sin embargo, un Diseño Basado en Prestaciones, donde lo que se requiere es alcanzar unos objetivos de seguridad y desempeño particulares, permitirá, en ocasiones, una racionalización económica importante sin reducir el grado de seguridad del edificio. No hay que olvidar que, en un entorno económico-social donde la optimización de los materiales y la reducción de los productos químicos utilizados en la construcción es de suma importancia, un Diseño Basado en Prestaciones permitirá en muchas ocasiones mejorar la eficiencia medioambiental del propio diseño.

El Diseño Basado en Prestaciones permite fijar objetivos que pueden ir encaminados, dependiendo de la propia normativa, a priorizar la seguridad de las personas o bien a incorporar además la conservación de los bienes materiales en caso de incendio.

En el caso del Código Técnico de la Edificación prevalece ante todo la seguridad de los ocupantes. Sin embargo, garantizar

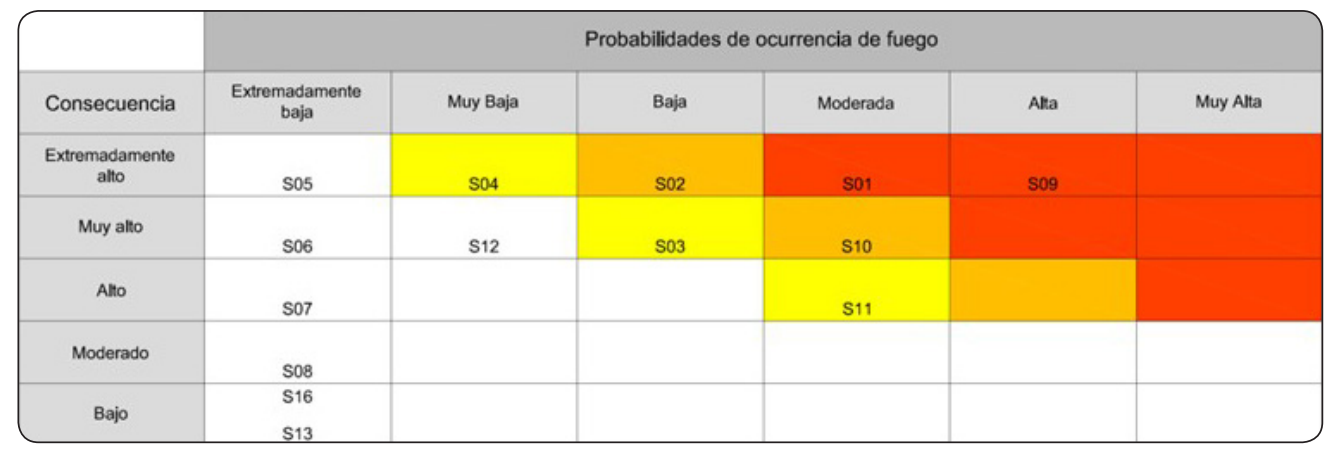

Figura 2. Matriz de probabilidad/daño utilizada habitualmente en la evaluación de los riesgos de incendio. 
el cumplimiento de sus seis exigencias básicas (propagación interior, propagación exterior, evacuación de ocupantes, instalaciones de protección contra incendios, intervención de los bomberos y resistencia al fuego de la estructura) permite minimizar el impacto económico que un posible incendio accidental tendrá en los edificios.

Cualquier edificio singular, donde el propio diseño arquitectónico planteado es incompatible con los requerimientos prescriptivos de seguridad frente a la acción del incendio, es susceptible de ser analizado mediante métodos basados en prestaciones. Los pabellones polideportivos, los mercados o los aeropuertos y las estaciones de transporte son algunos de los casos en los que afrontar la seguridad de la estructura a partir de métodos prestacionales tendrá unas ventajas muy significativas respecto del simple análisis prescriptivo.

Un campo de aplicación muy importante de los métodos prestacionales cuando se evalúa la seguridad de las estructuras en caso de incendio es la rehabilitación de edificios históricos. La utilización del Diseño Basado en Prestaciones permitirá adoptar aquellas medidas de seguridad más adecuadas que permitan garantizar la seguridad de las personas a la vez que se posibilita garantizar la imagen original del edificio catalogado.

\section{EVOLUCIÓN HISTÓRICA}

Los primeros pasos para la implementación de un método basado en prestaciones surgen en 1928, cuando Ingberg establece una relación de tiempo equivalente a partir de las áreas equivalentes que engloban, respectivamente, la curva normalizada y la curva real. Sin embargo, este método debe ser descartado. No existe ninguna correlación física o matemática que pueda apoyar este método de equivalencia. Debido a que el flujo de calor por radiación es directamente proporcional a la temperatura de la superficie emisora a la $4 .^{\mathrm{a}}$ potencia, las consecuencias de dos incendios con la misma área encerrada por la curva temperatura-tiempo, pero diferente potencia liberada, pueden ser completamente diferentes.

Es durante los años setenta cuando Law y Petterson definen lo que hoy en día se conoce como el método del tiempo equivalente (5) (6) (7) que, si bien no podemos considerarlo un método prestacional, sí que representa un avance en el tratamiento de las estructuras de acero protegido y de hormigón armado. Sin entrar en las particularidades del método, hay que indicar que el mismo ya tiene en cuenta aspectos particulares de cada edificio, como son el factor de ventilación de escenario, algo que Ingberg no había considerado, y la densidad total de carga de fuego.

Al mismo tiempo, entre 1970 y 1980 Magnusson y Thelandersson realizan un profundo análisis de las leyes térmicas que se producen en diversos escenarios, mediante el estudio del flujo de calor emitido por radiación en función de la potencia del incendio. Esto lleva a la definición de unas curvas temperatura-tiempo que definen el escenario de incendio después de producirse la combustión súbita generalizada, con combustibles de origen celulósico y para diversos coeficientes de ventilación (8).

Este estudio permite obtener lo que se puede definir como unas curvas temperatura-tiempo de fuegos reales analíticos, pero que posteriormente ha sido contrastado con un gran nú- mero de ensayos de laboratorio y donde se ha obtenido una correlación de resultados excelente. Estas curvas han sido adoptadas, por ejemplo, por la normativa sueca, y permiten evaluar de manera simplificada, conociendo la ventilación y la densidad total de carga de fuego, cuáles son las temperaturas a las que se verán sometidos los elementos estructurales en caso de incendio.

Con el mismo objetivo de determinar cuáles son las temperaturas reales que afectan a una estructura en caso de incendio Wickström, durante el periodo 1980-1981, define las curvas de fuego paramétricas, utilizando las curvas de Magnusson y Thelanderson como elemento de contraste para su validación. Estas curvas hay que decir que no tienen como objetivo la definición de una ley real de temperaturas en función del tiempo, sino que su objetivo es poder dictaminar sobre las máximas temperaturas a las que se verán sometidos los elementos estructurales en caso de incendio. Las limitaciones del método son importantes. Por ejemplo, no se pueden utilizar en sectores de incendio superiores a los $500 \mathrm{~m}^{2}$, ni en alturas mayores de $4 \mathrm{~m}$. Sin embargo, cumpliendo estas condiciones y siendo conscientes que únicamente se pueden utilizar en el caso de analizar incendios de origen celulósico, los resultados obtenidos representan una buena aproximación a la realidad.

Hasta ahora todos los métodos que se han presentado son analíticos, y permiten obtener de manera simplificada las temperaturas que se producen en un escenario de incendio. Sin embargo, a la vez que durante los años sesenta se producen investigaciones relativas a los balances energéticos en los incendios (9) (10) y durante los años setenta una serie de acontecimientos promueve el cambio de las normativas hacia un enfoque claramente prestacional, a nivel tecnológico, también se producen una serie de avances que permitirían el estudio preciso de las consecuencias de un incendio en un edificio concreto. El año 1976 Petterson, Magnusson y Thor exponen con claridad las ecuaciones de balance energético que definen claramente el comportamiento del incendio en espacios cerrados y desarrollan los primeros modelos informáticos de zona, gracias a los cuales se permite analizar de manera particular las leyes temperatura-tiempo que acontecen en un escenario (11). Teniendo en cuenta parámetros como la geometría del escenario, las características de los cerramientos y las propiedades del combustible y el incendio se pueden obtener las temperaturas en los gases calientes, considerando que tanto la temperatura, la densidad, la energía y la presión del gas son homogéneas en todo el recinto del incendio.

A lo largo de los años estos modelos de zona han evolucionado hacia los modelos de dos zonas, pudiendo así diferenciar capas superiores de los gases calientes y capas inferiores de los gases fríos. Esto ha permitido estudiar de manera simplificada el grado de seguridad no sólo de la estructura, sino también de las vías de evacuación, de cara a la seguridad de los ocupantes.

Actualmente son las simulaciones basadas en la dinámica computacional de fluidos (CFD), las teorías de la termodinámica y las de la combustión las que permiten evaluar con un mayor grado de precisión cuáles son las temperaturas que inciden en los elementos estructurales. Estas simulaciones resuelven las ecuaciones diferenciales parciales de NavierStokes para gases compresibles, permitiendo evaluar varia- 
bles como la temperatura, la densidad, la velocidad de los gases o la concentración de especies químicas en función del espacio y del tiempo. Esto, unido a las teorías de la Mecánica de los Medios Continuos, la Resistencia de los Materiales y el método de los elementos finitos permite analizar con un nivel de detalle muy alto el comportamiento de las estructuras en caso de incendio.

Se puede apreciar, con este breve recorrido histórico desde los años 1920 hasta la actualidad (Figura 3), como el objetivo siempre ha sido poder garantizar la seguridad de las personas (y en paralelo evaluar el grado de seguridad real de las estructuras) a través del diseño prestacional, particularizando las curvas temperatura-tiempo concretas para cada edificio.

\section{MARCO NORMATIVO NACIONAL E INTERNACIONAL}

Una cada vez más importante demanda de flexibilidad por parte de los arquitectos en el diseño de las construcciones, así como la rápida evolución de las tecnologías de la edificación, ha promovido que desde el año 2006 el Código Técnico de la Edificación (1) haya promovido la utilización del Diseño Basado en Prestaciones, es decir, la incorporación de soluciones alternativas con prestaciones equivalentes a las exigencias básicas del Código Técnico.

Este carácter prestacional del diseño de las estructuras frente a la acción del fuego tiene a día de hoy un breve recorrido en España, escasamente diez años. Sin embargo, existen acontecimientos en diferentes países desde los años setenta (12) que vienen promoviendo esta metodología de trabajo. Países como el Reino Unido, Japón, Estados Unidos, Australia o Nueva Zelanda se pueden considerar precursores en la introducción de los métodos prestacionales en sus códigos de protección frente al fuego de las estructuras. En todos estos países se realizaron importantes esfuerzos para adaptar sus códigos prescriptivos durante las décadas de los años setenta y ochenta.

Un importante ejemplo de ello es el Reino Unido, donde los Códigos de Edificación habían tenido una importante compo- nente prescriptiva con el objetivo de evitar incendios como el que destruyó Londres el año 1666. Sin embargo, unas reglas prescriptivas cada vez más costosas de aplicar, tanto a nivel técnico como económico, promueven un cambio en el marco legislativo, fomentando el año 1985 un Código basado en Prestaciones.

A modo de ejemplo, la guía NFPA 101, Código de Seguridad Humana (13), contiene en su capítulo 5 lo que denomina el Diseño Basado en Desempeño. En éste no se especifican reglas concretas para llevar a cabo el diseño, sino que se establecen unas metodologías y unos objetivos de desempeño que deberán ser evaluados a partir del diseño propuesto. En esta importante guía americana se recogen algunos aspectos que deben ser básicos en cualquier diseño basado en prestaciones. En primer lugar establece en ocho el número mínimo de escenarios de incendio que deben ser analizados (Tabla 1), a no ser que las características particulares del edificio determinen que un número inferior de escenarios son suficientes para garantizar la seguridad de los ocupantes.

Una de las últimas incorporaciones al marco normativo internacional de los métodos prestacionales es un anejo, no mandatorio, titulado «Performance-based Design Procedures for Fire Effects on Structures» que será publicado en la nueva versión de la ASCE-7, Minimum Design Loads and Associated Criteria for Buildings and Other Structures (14). Este anejo E aporta los procedimientos para la evaluación prestacional de los elementos estructurales y sus conexiones. Esto constituye una clara alternativa al diseño tradicionalmente prescriptivo para alcanzar los requerimientos de diseño de la estructura.

De manera complementaria a todo este marco normativo internacional, actualmente existe un conjunto de documentos considerados fundamentales en el desarrollo de los estudios prestacionales. Se trata del conjunto de normas publicadas por el Comité Técnico internacional ISO TC 92/SC-4 «Fire safety». Dichas normas están siendo actualmente traducidas como normas UNE-ISO españolas por el Comité Técnico de Normalización 23 de AENOR, Subcomité 8 «Ingeniería de protección contra incendios» (AEN/CTN23/SC8).
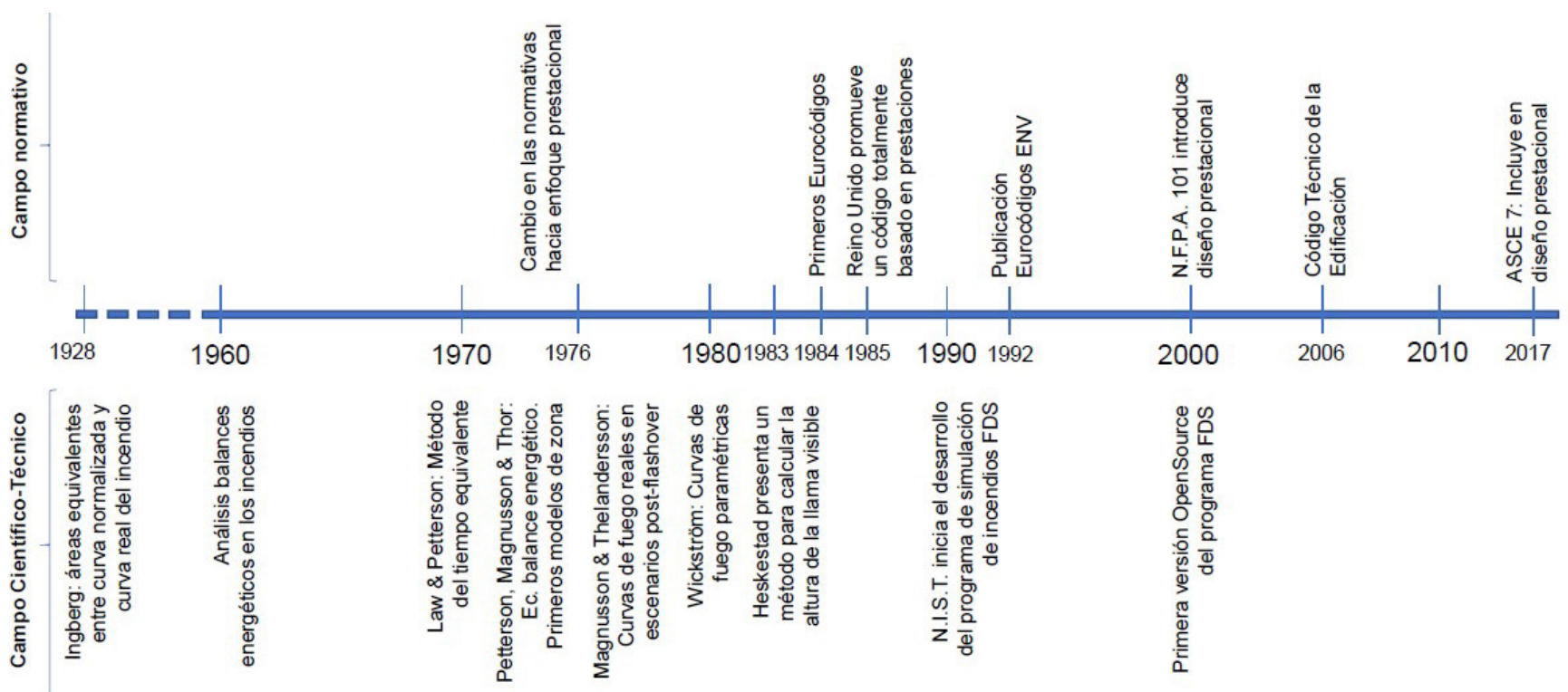

Figura 3. Línea del tiempo de los principales hitos en la implementación del Diseño Basado en Prestaciones. 
Tabla 1. Número mínimo de escenarios a considerar según la NFPA 101 (13).

\begin{tabular}{|c|l|}
\hline $\begin{array}{c}\text { Escenario } \\
\text { de incendio }\end{array}$ & \multicolumn{1}{c}{ Descripción del escenario, extraído de la NFPA 1o1 } \\
\hline 1 & $\begin{array}{l}\text { Escenario para el diseño específico de la ocupación, representativo del número y tipo de ocupantes. Tendrá en cuenta } \\
\text { la actividad, ocupación, tamaño de estancias, contenido, combustibles y ventilación }\end{array}$ \\
\hline 2 & $\begin{array}{l}\text { Incendio de desarrollo ultra-rápido, con las puertas interiores abiertas al iniciarse el incendio. Incluye las consecuencias } \\
\text { en la reducción de las vías de evacuación }\end{array}$ \\
\hline 3 & $\begin{array}{l}\text { Inicio del incendio en una habitación vacía que pone en riesgo a un espacio próximo con gran número de ocupantes por } \\
\text { crecimiento del fuego }\end{array}$ \\
\hline 4 & $\begin{array}{l}\text { Inicio del incendio en un espacio confinado de pared o falso-techo adyacente a una sala grande ocupada. El incendio } \\
\text { incluirá las consecuencias de no disponer sistema de detección ni supresión allí donde se origina el incendio }\end{array}$ \\
\hline 5 & $\begin{array}{l}\text { Incendio que se desarrolla lentamente, muy próximo a un área ocupada y donde no llegan los sistemas de supresión } \\
\text { automática. Fuente de ignición pequeña que ocasiona un incendio importante }\end{array}$ \\
\hline 6 & $\begin{array}{l}\text { Incendio más severo resultante de la máxima carga combustible que el edificio puede contener en un uso habitual } \\
\text { del mismo. Incendio de desarrollo rápido con los ocupantes }\end{array}$ \\
\hline 7 & $\begin{array}{l}\text { Incendio de exposición exterior, que se produce en una ubicación alejada del área de estudio, pero que bloquea las vías } \\
\text { de evacuación o se desarrolla en condiciones inaceptables }\end{array}$ \\
\hline 8 & Incendio con combustibles habituales en un espacio donde fallan todos los sistemas contraincendios \\
\hline
\end{tabular}

\section{EL DISEÑO BASADO EN PRESTACIONES APLICADO AL PABELLÓN DEPORTIVO DE LA MAR BELLA}

Un caso de estudio donde BAC Engineering Consultancy Group ha recurrido al Diseño Basado en Prestaciones para garantizar la integridad de la estructura en caso de incendio es el pabellón deportivo de La Mar Bella, situado en el barrio del Poblenou de Barcelona.

El pabellón tiene una volumetría que resulta de la unión de dos cuerpos superpuestos. El volumen situado en la parte inferior, el zócalo, es el de mayores dimensiones en planta, aproximadamente $100 \times 80 \mathrm{~m}$, mientras que el volumen superior tiene unas dimensiones de aproximadamente $80 \times 56$ $\mathrm{m}$, con una altura libre desde la pista hasta la cota inferior de la estructura de cubierta de aproximadamente 11,90 m.

Las fachadas longitudinales están realizadas en su totalidad con un cerramiento de vidrio laminado de $6+6 \mathrm{~mm}$ de grosor. Las fachadas de los testeros tienen vidrio únicamente hasta 2,5 m de altura, siendo el cerramiento de paneles opacos de madera laminada a partir de esa altura.

La estructura principal del pabellón se organiza en una serie de pórticos separados 3,75 m, con una luz máxima entre pilares de unos $49 \mathrm{~m}$. La estructura vertical está formada por pantallas de hormigón armado, con unas dimensiones de $3,75 \times 0,30 \mathrm{~m}$. La estructura horizontal de la cubierta se realiza mediante unas cerchas metálicas de aproximadamente 2,85 m de canto y una luz de unos $49 \mathrm{~m}$. El tipo de unión de estas cerchas con los pilares de hormigón es de gran relevancia para el estudio prestacional desarrollado. Éstas apoyan en las pantallas de hormigón mediante su cordón superior, sobre apoyos de neopreno armado de $30 \mathrm{~mm}$ de espesor, dejando el cordón inferior libre. Para asegurar la posible dilatación térmica debido a acciones climáticas ordinarias los apoyos de neopreno y los montantes extremos permiten la libre dilatación de $25 \mathrm{~mm}$ en cada lado de la cercha (Figura 4).

Existen cuatro franjas horizontales de arriostramiento, encargadas de estabilizar el plano superior de la cubierta.

El cerramiento de la cubierta está formado por una chapa ondulada sobre la que se apoya un panel aislante y 50-70 mm

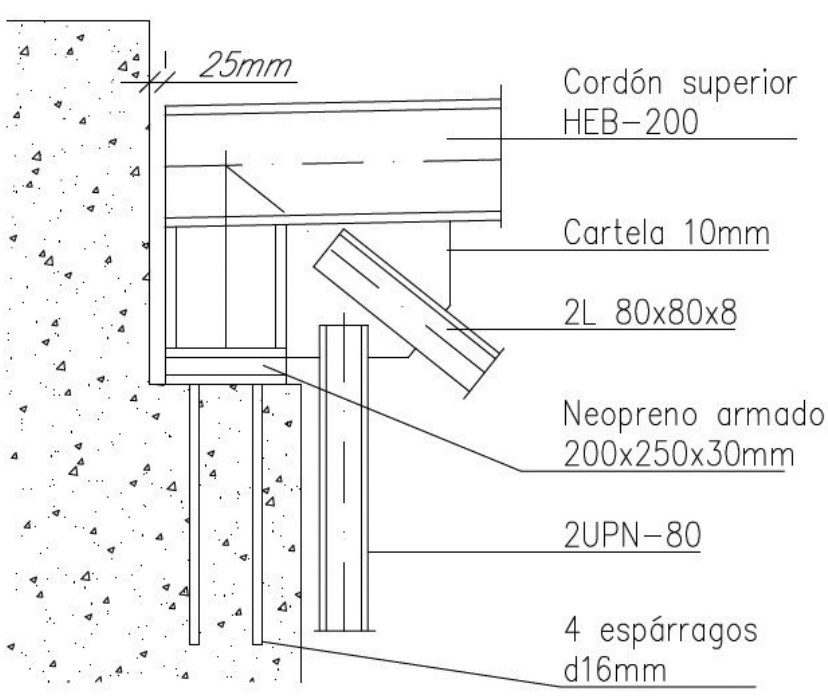

Figura 4. Detalle de apoyo de la cercha de cubierta en las pantallas de hormigón armado, mediante neopreno armado.

de gravas. Teniendo en cuenta que el peso de las cargas permanentes es superior a $1 \mathrm{kN} / \mathrm{m}^{2}$, según los criterios del CTE no se puede considerar que se trate de una cubierta ligera, debiendo garantizar en tal caso una resistencia al fuego de 120 minutos, por las características del edificio y de las vías de evacuación, teniendo recorridos ascendentes.

El objetivo fundamental de este estudio prestacional es poder dictaminar sobre la necesidad de disponer un sistema de protección pasiva en la estructura y, por lo tanto, de su posterior mantenimiento cada cierto periodo de tiempo, dependiendo del producto de protección utilizado. Teniendo en cuenta las condiciones de apoyo de las cerchas en las pantallas de hormigón armado, se puede producir una inversión de los esfuerzos habituales dependiendo del incremento térmico generado durante un posible incendio accidental; el esfuerzo de tracción del cordón inferior puede cambiar a compresión si la dilatación longitudinal de la cercha es superior a los 25 + $25 \mathrm{~mm}$ que las condiciones de apoyo permiten desplazar. De la aplicación de la ecuación que define la elongación de un elemento lineal sometido a incrementos térmicos [2], particularizada para el material acero y los datos del proyecto, se obtiene que un incremento de temperatura superior a $85^{\circ} \mathrm{C}$ 
en toda la cercha es suficiente para generar la inversión de esfuerzos.

$$
\Delta l=\Delta \cdot l \cdot \alpha
$$

Se establecen cuáles son los riesgos de incendio más probables del edificio y cuáles son los escenarios de fuego que de estos riesgos se derivan. Consensuados éstos con el cliente, se acuerda considerar que pueden producirse usos diferentes a los puramente deportivos, como, por ejemplo, que se instale un escenario en la pista polideportiva. Un análisis preliminar determina cinco riesgos de incendio como probables, siendo dos de ellos los que más pueden afectar a la estructura principal de la cubierta (Tabla 2).

Para evaluar con precisión cuáles son las temperaturas obtenidas en cada uno de estos dos escenarios, considerados críticos, se llevan a cabo una serie de simulaciones basadas en la dinámica computacional de fluidos (CFD), analizando los procesos de combustión y transferencia de calor, considerando las ecuaciones de Navier-Stokes adaptadas a flujos compresibles a baja velocidad. Se utiliza el programa desarrollado específicamente en el NIST (15) para este tipo de simulaciones de incendio, el Fire Dynamics Simulator (FDS).

El estudio de cada uno de estos escenarios debe incluir estudios de sensibilidad de los resultados obtenidos, garantizando así que posibles variaciones en datos inciertos no afectará a la seguridad de la estructura. Es por ello que se realizan simulaciones variando diferentes parámetros de entrada, como son la temperatura de rotura de los vidrios de fachada y la producción de partículas sólidas. Debido al gran volumen de aire analizado, superior a los $50.000 \mathrm{~m}^{3}$, se determina que variaciones en estos parámetros no tienen repercusión en las temperaturas obtenidas en la estructura de cubierta.

Del análisis matemático realizado se determina que es el incendio de una tarima de espectáculos en la pista principal el incendio más desfavorable de cara a la seguridad de la estructura. Las llamas alcanzan una altura media de $7 \mathrm{~m}$ (Gráfico 1), identificando éstas con temperaturas de los gases superiores a los $500-520^{\circ} \mathrm{C}$. En el penacho del incendio los valores térmicos a la altura de la estructura alcanzan los $350^{\circ} \mathrm{C}$, mientras que el resto de la estructura se ve sometida a temperaturas medias de $100-150^{\circ} \mathrm{C}$, correspondientes a los valores térmicos de la capa de humos, tal y como se aprecia en la Figura 5 (a).

La temperatura media de los gases calientes, fuera del ámbito del penacho, se mantiene comprendida entre los $50^{\circ} \mathrm{C}$ y los $100^{\circ} \mathrm{C}$, excepto en la zona de la capa de humos situada en la parte superior del volumen. En este ámbito superior los valores térmicos ascienden hasta los $100^{\circ} \mathrm{C}$ y los $150^{\circ} \mathrm{C}$, según se aprecia en la Figura 5 (b).

Con el objetivo de realizar posteriormente modelos termomecánicos de la estructura de cubierta FDS incluye el cálculo de las temperaturas superficiales adiabáticas (AST), parámetro que mide el flujo de calor en una superficie sólida. Mediante el concepto expuesto por Wickström (16) la tempera-

Tabla 2. Escenarios considerados y estudios de sensibilidad asociados.

\begin{tabular}{|c|l|c|l|}
\hline Escenario & \multicolumn{1}{|c|}{ Descripción } & \multicolumn{1}{|c|}{$\begin{array}{c}\text { Potencia } \\
\text { liberada }\end{array}$} & \multicolumn{1}{c|}{ Estudios de sensibilidad } \\
\hline 1 & $\begin{array}{l}\text { Tarima de espectáculos en pista } \\
\text { principal }\end{array}$ & $625 \mathrm{~kW} / \mathrm{m}^{2}$ & $\begin{array}{l}\text { Variación de la producción de partículas sólidas } \\
\text { Variación en la temperatura de rotura de los vidrios de fachada }\end{array}$ \\
\hline 2 & $\begin{array}{l}\text { Fuego en despachos } \\
\text { administrativos del altillo }\end{array}$ & $500 \mathrm{~kW} / \mathrm{m}^{2}$ & $\begin{array}{l}\text { Variación de la producción de partículas sólidas } \\
\text { Variación en la temperatura de rotura de los vidrios de fachada }\end{array}$ \\
\hline
\end{tabular}

Temperaturas en el penacho

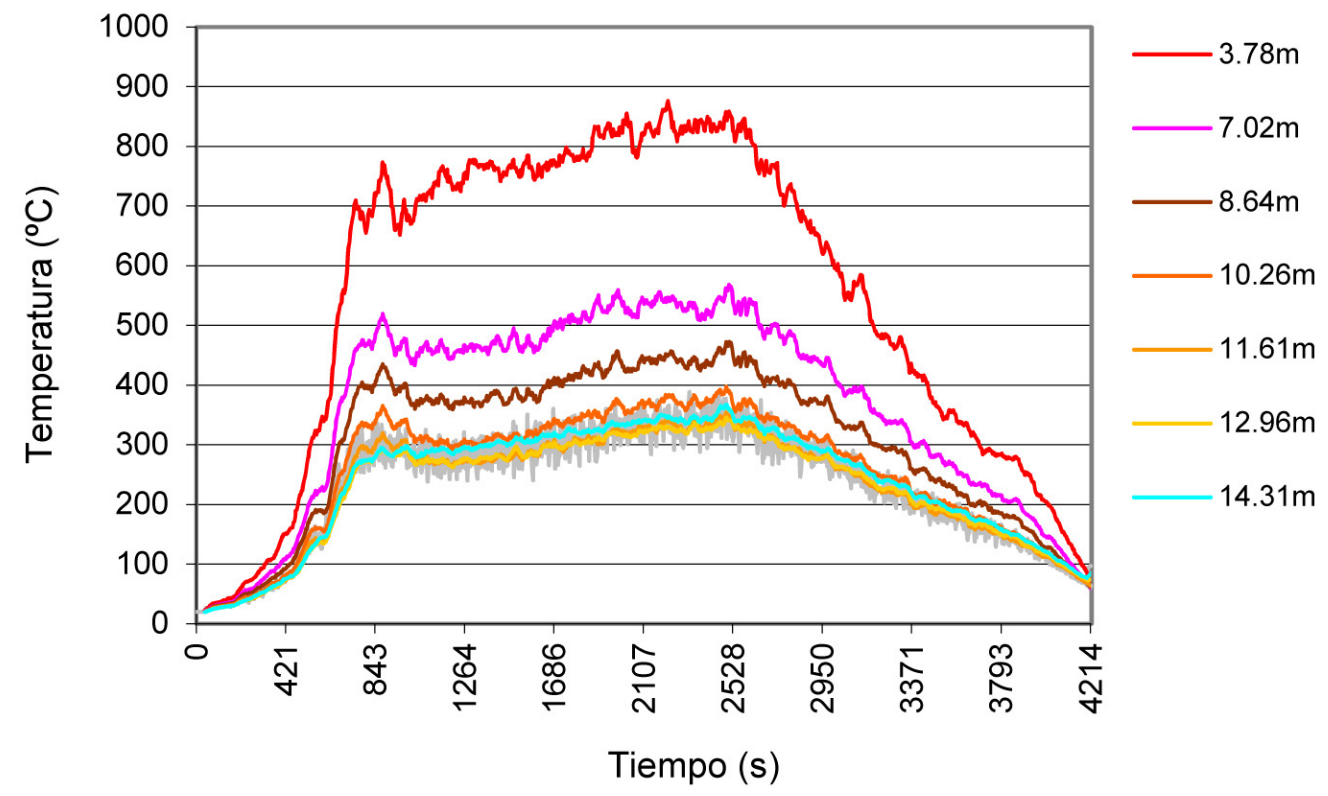

Gráfico 1. Valores temperatura-tiempo en función de la altura en el penacho del incendio. 
a)

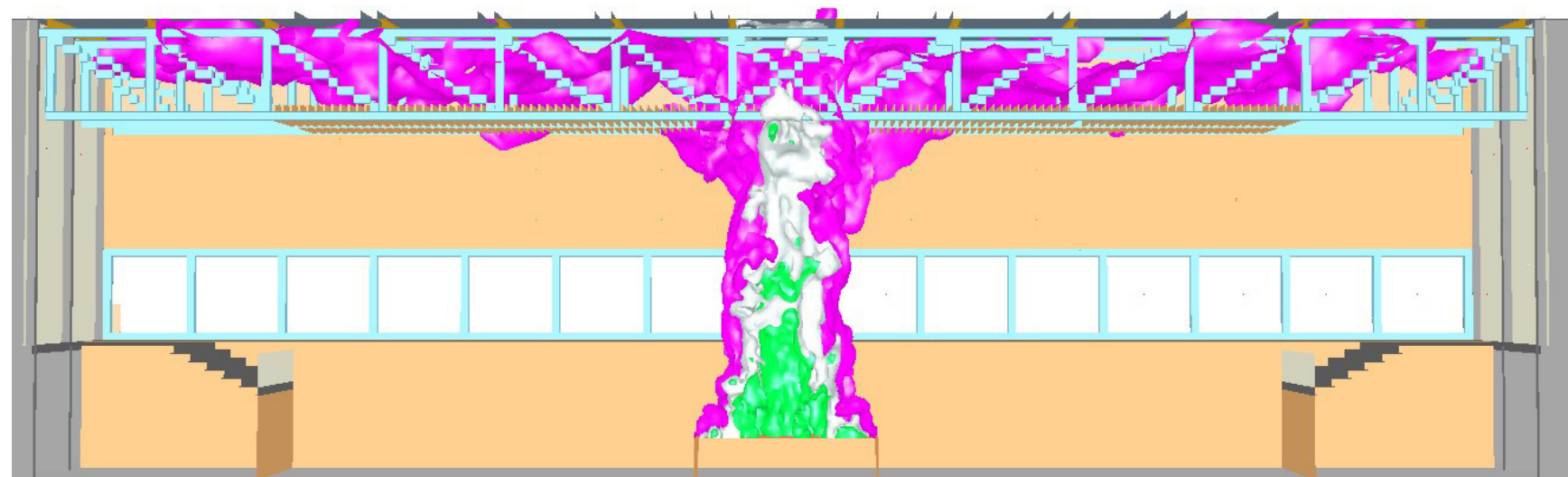

b)
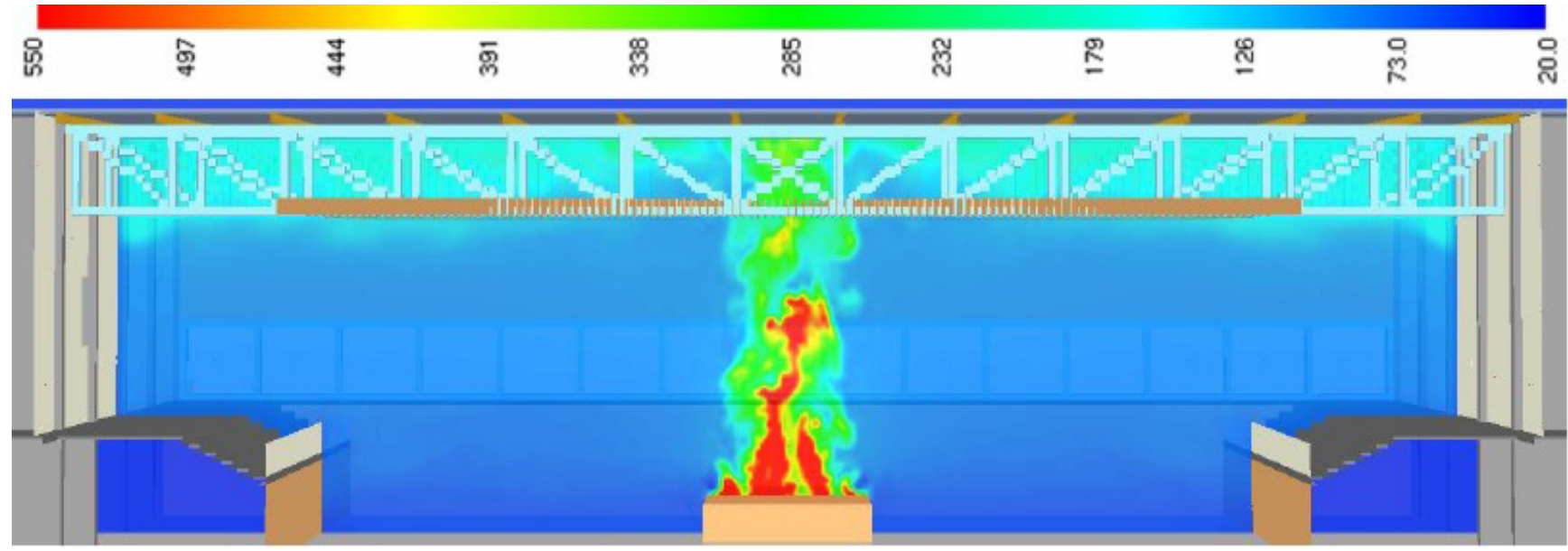

Figura 5. (a) Iso-superficies térmicas $\left(150,300\right.$ y $\left.550^{\circ} \mathrm{C}\right)$ durante la fase estacionaria del incendio y (b) distribución de temperaturas en los gases calientes durante el desarrollo del incendio. Corte transversal.

tura adiabática en una superficie sólida es aquella para la cual el flujo de calor neto es nulo [3].

$$
q_{\text {net }}^{\prime \prime}=\varepsilon_{s} \cdot\left(q_{\text {inc }, \text { rad }}^{\prime \prime}-\sigma \cdot T_{s}^{4}\right)+h \cdot\left(T_{g a s}-T_{s}\right)=0
$$

Siendo $\varepsilon_{s}$ la emisividad de la superficie (17), $h$ coeficiente de transferencia de calor por convección, $q_{\text {inc,rad }}^{\text {"r }}$ el flujo de calor por radiación incidente en la superficie, y $T_{\text {gas }}$ la temperatura del gas que envuelve el sólido.

El cordón inferior de la cercha se ve sometido a unos valores próximos a los $380^{\circ} \mathrm{C}$ en el penacho del incendio y $100-120^{\circ}$ $\mathrm{C}$ en el resto del cordón inferior (Gráfico 2).

Una vez definidas las temperaturas en la estructura (Figura 6) se lleva a cabo un modelo de análisis termo-mecánico, con el fin de poder dictaminar sobre el comportamiento del entramado resistente cuando acontece un incendio accidental.

Se determina que el cordón superior tiene un alargamiento de $75 \mathrm{~mm}$. El cordón inferior se ve sometido a desplazamientos de dos naturalezas diferentes. El primero se deriva de la propia dilatación longitudinal de la estructura. El segundo se produce como consecuencia del giro de los montantes extremos debido a la deformación vertical de la estructura. Esto implica que el desplazamiento total del cordón inferior sea de $106 \mathrm{~mm}$, superior a los $50 \mathrm{~mm}$ para los que están diseñados los apoyos laterales de neopreno. Dadas las condiciones de apoyo (Figura 4), el modelo estructural debe recoger los esfuerzos que se deriven de la dilatación longitudinal que queda coartada por la propia condición de contorno de la estructura, es decir, $56 \mathrm{~mm}$.

Este modelo estructural recoge las no linealidades mecánicas, es decir, los cambios en las propiedades mecánicas del acero (17) y (18) cuando se ve sometido a altas temperaturas. Aunque se considera que su capacidad resistente no se ve reducida hasta $\operatorname{los} 400^{\circ} \mathrm{C}$, su límite de proporcionalidad y su módulo de elasticidad sí que se ven afectados a partir de $\operatorname{los} 100^{\circ} \mathrm{C}$.

En el momento en que la estructura se dilata $50 \mathrm{~mm}$ y el movimiento longitudinal queda coartado, algo que sucede aproximadamente cuando los gases calientes acumulados en la capa de humos alcanzan un valor estacionario igual o superior a los $85^{\circ} \mathrm{C}$, se invierten los esfuerzos de la estructura, produciéndose una inestabilidad geométrica por compresión del cordón inferior HEB-120, con una longitud libre de $49 \mathrm{~m}$.

Teniendo en cuenta los condicionantes arquitectónicos y la configuración del falso techo discontinuo del pabellón se excluye como posible protección pasiva cualquier tipo de mortero o vermiculita. Las pinturas intumescentes, como único sistema pasivo posible, reaccionan a los incrementos térmicos superiores a los $200^{\circ} \mathrm{C}$ aproximadamente. El análisis prestacional determina que el colapso de las cerchas de cubierta se puede llegar a producir para unas temperaturas de la estructura inferiores al valor de reacción del producto químico, por lo 


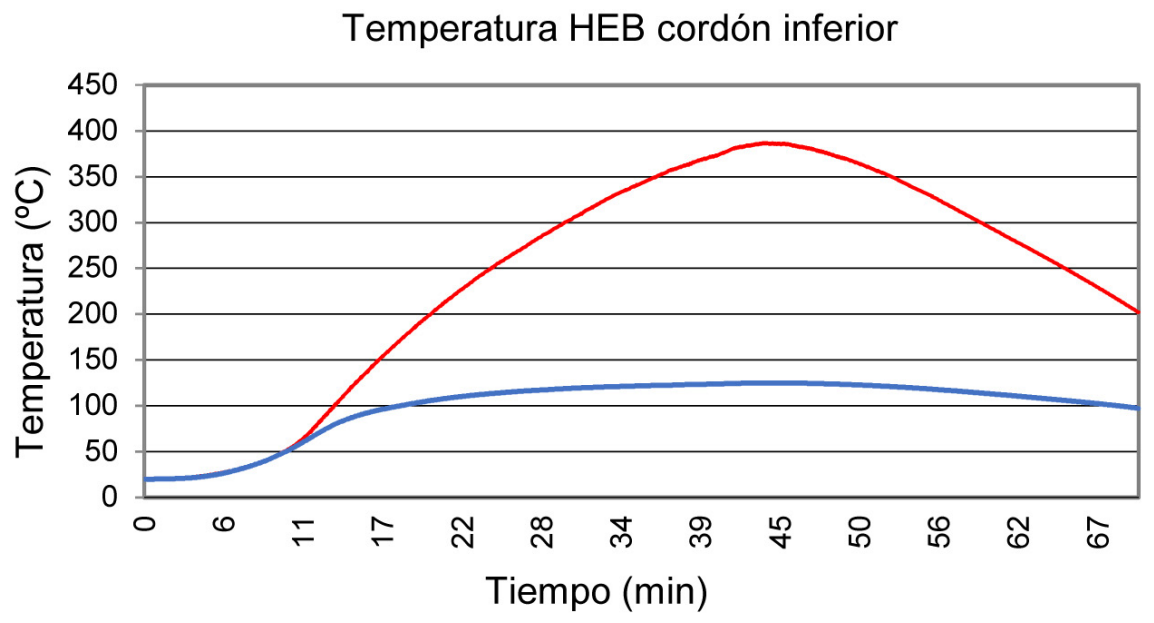

Gráfico 2. Temperaturas adiabáticas en el cordón inferior de la estructura principal, diferenciando la zona del penacho y la de la capa de humos.

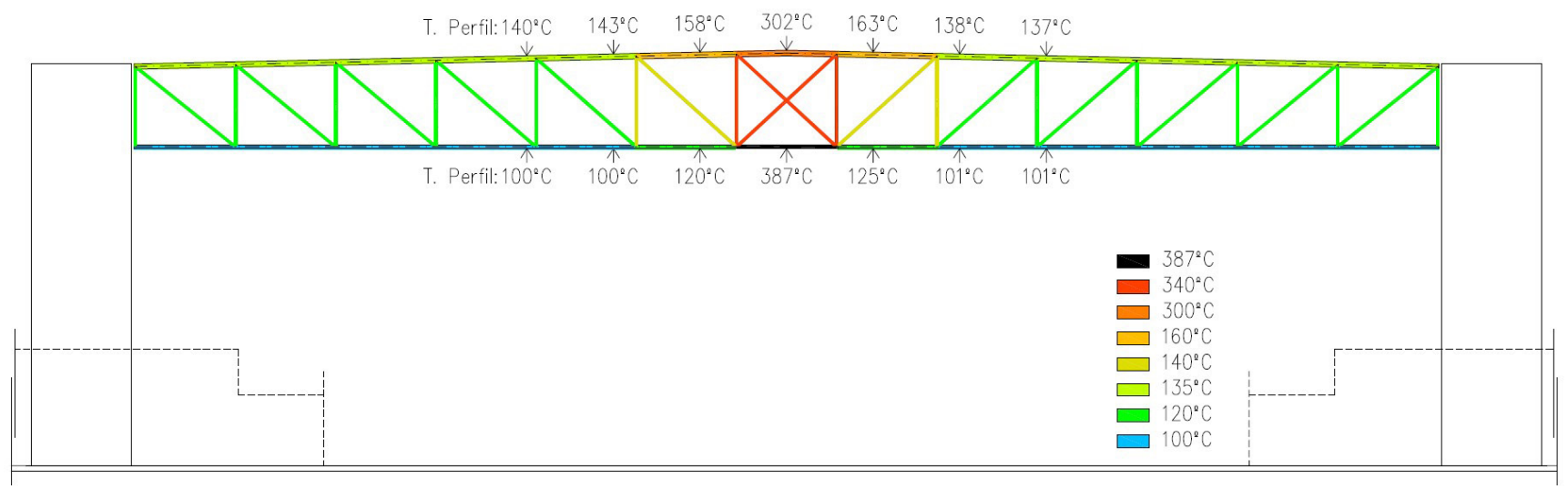

Figura 6. Distribución de temperaturas máximas obtenidas en la cercha alineada con el penacho del incendio.

que en este caso particular una inversión económica en la aplicación de una pintura intumescente no garantiza la seguridad de la estructura en caso de incendio accidental.

En este punto existen dos soluciones para garantizar la seguridad de la estructura principal del pabellón. En primer lugar se puede recurrir a la instalación de sistemas activos de protección, tales como un sistema de control de temperatura y evacuación de humos (19), capaces de limitar la temperatura de la capa de humos a $80^{\circ} \mathrm{C}$. Sin embargo, en este caso particular, la seguridad del entramado resistente también se puede garantizar actuando sobre el comportamiento de la estructura en caso de incendio, evitando que se produzca la inversión de esfuerzos en el cordón inferior de las cerchas principales.

Finalmente, habiendo valorado las repercusiones arquitectónicas, de plazo de ejecución y de coste de ambos posibles caminos, se opta por actuar sobre el comportamiento de la estructura en caso de incendio. Para lograrlo se realizan dos cortes de $30 \mathrm{~mm}$ de ancho, uno en cada extremo de la cercha, en el último vano del cordón inferior, que es capaz de absorber el movimiento producido por la dilatación en caso de incrementos térmicos, sin que ello afecte al comportamiento del elemento estructural (Figura 7). Este corte afecta tanto al

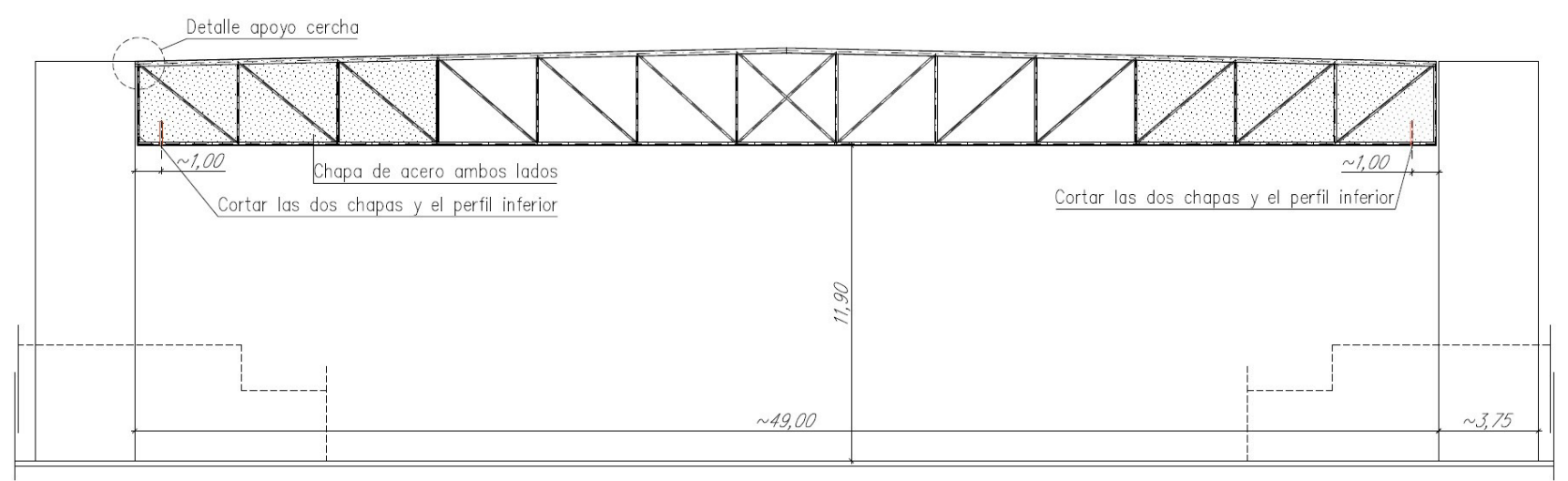

Figura 7. Modificación de la respuesta de la estructura principal en caso de incendio. 
cordón inferior como a las dos chapas de acero existentes a cada lado de la cercha.

\section{CONCLUSIONES}

El presente artículo muestra la capacidad del Diseño Basado en Prestaciones cuando se debe afrontar la seguridad de las estructuras de edificación frente a la acción del fuego. Se realiza un recorrido histórico por el método, mostrando los avances que se han producido en las últimas décadas en esta materia de estudio.

La utilización del Diseño Basado en Prestaciones permite conocer cuál es el comportamiento real de una estructura, por singular que ésta sea, frente a la acción del fuego. Este conocimiento relativo al comportamiento real de la estructura en caso de incendio permite al diseñador adoptar aquellos sistemas de seguridad más adecuados en cada caso particular.

Este conocimiento detallado también permite tener una mayor flexibilidad en la fase de diseño frente a la aplicación de los métodos prescriptivos. Con el objetivo de mostrar las ventajas que este conocimiento real de la respuesta estructural aporta al diseñador se muestra finalmente un caso de estudio real, en el que se verifica que la aplicación de los métodos prescriptivos no siempre es capaz de detectar comportamientos singulares de la estructura en caso de incendio. En este caso particular la protección mediante pinturas intumescentes no hubiese solucionado la integridad de la estructura en caso de incendio.

\section{AGRADECIMIENTOS}

Se quiere agradecer al Ayuntamiento de Barcelona (Institut Barcelona Esports) la posibilidad de mostrar detalladamente los resultados obtenidos en el Diseño Basado en Prestaciones de la estructura principal del Pabellón de La Mar Bella en caso de incendio. También se quiere agradecer a todos los miembros de BAC Engineering Consultancy Group que han colaborado en la redacción del estudio prestacional del pabellón de La Mar Bella.

\section{REFERENCIAS}

(1) Ministerio de Fomento (2006). Código Técnico de la Edificación. Parte 1.

(2) AENOR (2004). UNE-EN 1991-1-2:2004 Eurocódigo 1: Acciones en estructuras. Parte 1-2: Acciones generales - Acciones en estructuras expuestas al fuego.

(3) Gann, R., Babrauskas, V., Peacock, R. (1994). Fire conditions for Smoke Toxicity Measurement. Fire and Materials, 18: 193-199.

(4) IFEG (International Fire Engineering Guidelines) (2005). Australian Building Codes Board. ISBN 1741614562.

(5) Pettersson, O. (1971). The possibilities of predicting the fire behaviour of structures on the basis of data from standard fire resistance tests. En Colloque sur les Principes de la Sécurité au Feu des Structures à Paris.

(6) Law, M., Arnault, P. (1972). Fire loads, Natural fires and standard fires. En ASCE-labse International Conference of Planning and Design of Tall Buildings. Conference Preprints: Reports Vol. 1b-8. Lehigh University, Pennsylvania.

(7) Pettersson, O. (1973). The connection between a real fire exposure and the heating conditions according to standard fire resistance Tests - with special application to steel structures. En European Convention for Constructional Steelwork, Doc. CECM 3-73/7E

(8) Magnusson, S. E., Thelandersson, S. (1970). Temperature-Time curves of complete process of fire development. Theoretical Study of Wood Fuel fires in Enclosed Spaces. Acta Polytechnica Scandinavica. Civil Engineering and Building Construction Series (65). Sweden: Lund Institute of Technology.

(9) Kawagoe, K., Sekine, T. (1963). Estimation of Fire Temperature-Time curve in rooms. Building Research Institute, Occasional Report, $n .^{\circ}$ 11. Tokyo.

(10) Ödeen, K. (1963). Theoretical study of fire characteristics in enclosed spaces. Bulletin (10). Stockolm: Division of Building Construction, Royal Institute of Technology.

(11) Pettersson, O., Magnusson, S. E., Thor, J. (1976). Fire engineering design of steel structures. Bulletin (52). Sweeden: SBI, Swedish Institute of Steel Construction, Lund Institute of Technology.

(12) Meacham, B. (1998). The evolution of Performance-Based Codes and Fire Safety design methods. National Institute of Standards and Technology.

(13) National Fire Protection Association. NFPA 101, Código de Seguridad Humana, edición 2015.

(14) ASCE 7-16 (2017). Minimum design loads and associated criteria for buildings and other structures, Appendix E.

(15) McGrattan, K., et al. (2016). Fire dynamics Simulator (version 6.5). User's Guide. National Institute of Standards and Technology (NIST) y VTT Technical Research Centre of Finland.

(16) Wickström, U., Duthinh, D., McGrattan, K. (2007). Adiabatic surface temperature for calculating heat transfer to fire exposed structures. En Proceedings of the Eleventh International Interflam Conference. Interscience Comunications.

(17) AENOR (2016). UNE-EN 1993-1-2:2016 Eurocódigo 3: Proyecto de estructuras de acero. Parte 1.2: Reglas generales. Proyecto de estructuras sometidas al fuego.

(18) Ortiz, J., Villa, J. (2009). Cálculo de las estructuras de acero frente al incendio. Editoriales APTA

(19) AENOR (2004). UNE 23585:2004 Seguridad contra incendios. Sistemas de control de temperatura y evacuación de humos (S.C.T.E.H.). Requisitos y métodos de cálculo y diseño para proyectar un sistema de control de temperatura y de evacuación de humos en caso de incendio. 